\title{
The Mechanics of Large-Volume-Change Transformations in High-Capacity Battery Materials
}

Matthew T. McDowell ${ }^{\mathrm{a}, \mathrm{b}, *}$, Shuman $\mathrm{Xia}^{\mathrm{a}, *}$, Ting $\mathrm{Zhu}^{\mathrm{a}, \mathrm{b}, *}$

${ }^{a}$ G. W. Woodruff School of Mechanical Engineering, Georgia Institute of Technology, Atlanta, GA, 30332, USA.

${ }^{b}$ School of Materials Science and Engineering, Georgia Institute of Technology, Atlanta, GA, 30332, USA.

*To whom correspondence should be addressed:

mattmcdowell@gatech.edu (M.T.M.)

shuman.xia@me.gatech.edu (S.X.)

ting.zhu@me.gatech.edu (T.Z.)

Keywords: Lithium-ion batteries, anode materials, stress, fracture, chemomechanics 


\section{ABSTRACT}

High-capacity next-generation materials for Li-ion and Na-ion batteries often undergo significant volume changes (up to $\sim 300 \%$ ) during reaction with Li or Na. These large-volumechange transformations cause mechanical fracture and pulverization of active battery materials, which can have detrimental effects on battery cycle life. Recent years have seen significant efforts dedicated to understanding the mechanics of such large-volume-change transformations in alloying anode materials. This review paperintroduces recent work focused on various aspects of the mechanics of alloying anode materials, includingin situ characterization of real-time reaction mechanisms and mechanical degradation processes, measurements of mechanical properties, measurements and simulations of spatiotemporal stress generation and evolution in active battery materials and structures, and studies on the interplay between chemistry and mechanics during reaction.In addition,mechanical effects across length scales within battery electrode structures are discussed. As demonstrated herein, the improved understanding of the mechanics of large-volume-change transformations has been essential for the rational design of durable high-capacity electrodes for Li-ion and Na-ion batteries. 


\section{INTRODUCTION}

Li-ion batteries are establishedas the most important energy storage technology for portable electronics and electric vehicles[1, 2]. Despite the success of Li-ion batteries, emerging applications such as low-cost electrified transportation and grid energy storage require batteries with improved performance characteristics and lower cost[1]. This necessitates the development of new high-capacity, low-cost electrode materials for Li-ion batteries[3-9], and/or the engineering of entirely new battery systems, such as lithium-sulfur, Na-ion, or flow batteries[1013]. Fundamental scientific and engineering challenges must be overcome in all these cases.

Conventional Li-ion batteries contain electrode materials that react with $\mathrm{Li}^{+}$ions via intercalation reactions. This reaction mechanism involves the insertion and removal of $\mathrm{Li}^{+}$ions as "guests" within the relatively invariant crystal structure of a host material, with only minor volumetric changes of the host ( 5-10\%)[14]. The small volume changes allow for reversibility over hundreds of cycles, and thus long cycle life. However, these materials have limited lithium storage capacity since the extra atoms within the host structure take up space within the electrode and contribute mass to the battery. A variety of materials are known that have much larger lithium storage capacities because they react via different reaction mechanisms, such as alloying and conversion reactions[15-17]. These reaction mechanisms are characterized by the formation of entirely new phases during reaction with $\mathrm{Li}^{+}$, and there is often significant (100-300\%) volume expansion/contractionduring cycling (Figure 1 inset). Such extreme volume changes in active material particles have traditionally led to poor cycle life for a number of reasons, including mechanical degradation/fracture of particles and side reactions accelerated by the continually changing surface area. Overcoming these issues would allow for the engineering of 
Li-ion batteries with higher energy density, and such progress would also enablenew materials for Na-ion batteries.

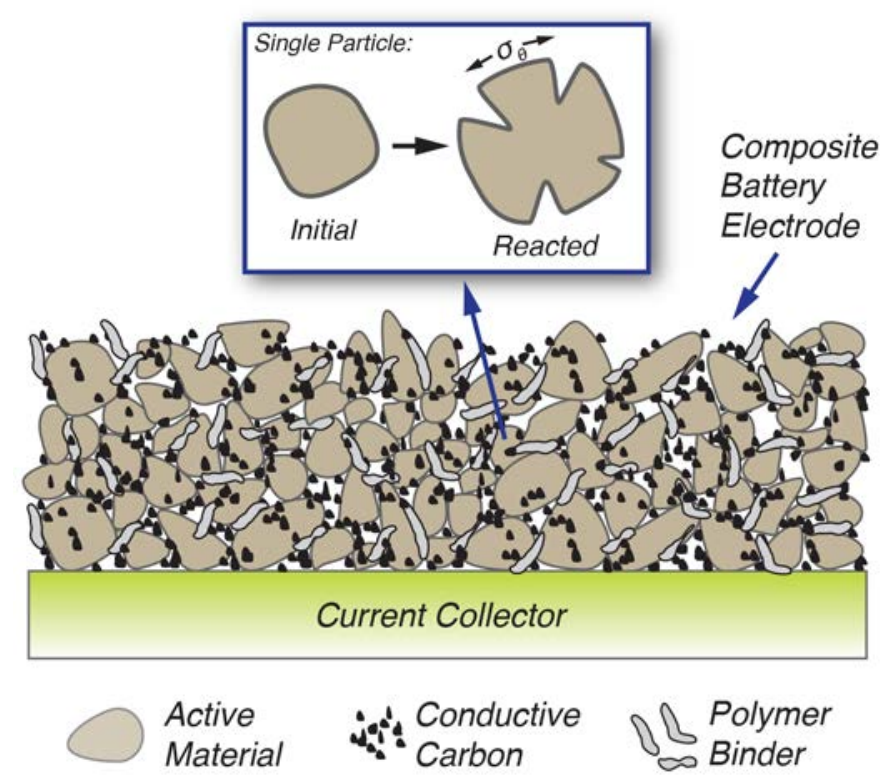

Figure 1. Schematic of a composite battery electrode containing particulate active material, conductive carbon, and polymeric binder. The inset shows a single active material particle before and after reaction with an alkali ion such as $\mathrm{Li}^{+}$or $\mathrm{Na}^{+}$; volume expansion and stress generation during reaction has caused cracks to form.

In recent years, it has become evident that the solid mechanics of alloying and conversion materials plays a major role in these extreme-volume-change transformations $[5,18]$. Transformation strains in individual active particles cause large stresses to exist within these materials due to ion concentration gradients or external/interfacial constraints. These stresses can cause fracture or void growth, and they lead to changes of morphology during cycling. Reactioninduced plasticity and flow are also important aspects of transformations in certain materials that influence the magnitude and spatial distribution of stresses within active material structures. Furthermore, evolved stresses are intrinsically linked to the thermodynamics and kinetics of the electrochemical reaction process via chemomechanical effects; this can have important ramifications for the energy efficiency during charge/discharge of a battery. Finally, large- 
volume-change materials also exhibit complex mesoscale interactions within battery electrodes: mechanical interactions between particles, as well as interfacial adhesion characteristics, play more important roles than in traditional materials.

Much recent groundbreaking work has been dedicated to fully understanding the mechanical aspects of electrochemical transformations in various alloying and conversion materials. This review paper is meant to provide an overview of recent experimental and modeling efforts in this area, with a focus on alloying reactions of anode materials.The review is organized in the following manner. First, experimental observations of volume changes and fracture processes in various materials willbe detailed. Then, studies on the mechanical properties and dynamic stress evolution within active material structures will be presented, along with the current understanding of the influence of stress on the thermodynamics and kinetics of reaction processes. Finally, recent work on the mesoscale and interfacial mechanics of electrode architectures (as schematically shown in Figure 1) will be discussed. Together, this body of work has provided much new knowledge regarding these unique transformations, and in many cases, fundamental mechanics insights have guided the development of materials with improved capacity and cycle life for Li-ion and Na-ion batteries [19, 20].

\section{OBSERVATIONS OF VOLUME CHANGE AND FRACTURE PROCESSES}

Understanding and controlling the mechanics of volume changes and fracture in battery materials is important for a multitude of reasons. Fracture of active particles during cycling can cause electrical disconnection of sections of the material from the electrode architecture, resulting in inactive "dead" material. Even when fracture does not occur, volumetricand morphological changes may also influence the electrical contact between particles. In addition, 
volume changes in anode materials influence the formation of the solidelectrolyte interphase (SEI) layer, a composite film that is formed on the surface of active particles due to electrolyte reduction. Volume change and fracture expose new surface area to the electrolyte, which results in the formation of additional SEI, consuming excess $\mathrm{Li}^{+}$ions and reducing the Coulombic efficiency during charge/discharge. Clearly, the control of volume changes and mechanical degradation is necessary for successful development of large-volume-change materials for batteries.

Much of the work to understand the mechanics of alloying battery materials has been motivated by experimental observations of volume changes and fracture. Many experimental studies on transformation mechanisms have been enabled by recent advances in in situ characterization methods, such as in situ transmission electron microscopy (TEM) and in situ Xray tomography[21-38]. Such experiments can reveal transformation processes in individual particlesin real time, andthis information can then be used to accurately model stress evolution and its effect on reaction processes. In this section, experimental observations of volume changes and fracture in various alloying materials will be introduced, setting the stage for further discussion of mechanical properties and stress evolution in later sections.

A variety of in situ and ex situ methods have been used to reveal volume changes and fracture processes in single nanostructures. High-capacity alloying anodes materials such as $\mathrm{Si}$, Ge, and Sn have been widely studied; Si has received the most attention due to its highest theoretical capacity and low cost. A "two-phase" reaction has been observed during the lithiation of crystalline Si nanostructures, with a highly lithiated phase $\left(\sim \mathrm{Li}_{3.75} \mathrm{Si}\right)$ separated from the crystalline Si by a reaction front of nanoscale thickness[39]. Crystalline Si nanoparticles and nanowires fracture during lithiation-induced volume expansion, with cracks initiating at the 
surface of particles[24, 25, 30, 40, 41]; this observation indicates that tensile hoop stress arises at the surface during lithiation.In situ TEM images oflithiation and fractureof a single Si particle areshown in Figure 2a-d. Particles smaller than $150 \mathrm{~nm}$ in diameter do not fracture, however, as the total strain energy in smaller particles is evidently not sufficient to initiate and drive crack growth [24]. In addition to these phenomena, the initial lithiation of crystalline Si has been shown to be highly anisotropic, with $\{110\}$ crystallographic planes reacting and expanding preferentially[25, 42-44]. This also influences fracture, as radial cracks tend to initiate at points of stress concentration at the surface between planes of preferential reaction[40]. After the initial lithiation/delithiation of crystalline $\mathrm{Si}$, the material transforms to the amorphous phase and remains amorphous with cycling[5]. Amorphous Si is more robust towards fracture, with a critical fracture size greater than $2 \mu \mathrm{m}[21,28,45]$. This has been attributed to the isotropic volume change observed for amorphous Si, which avoids the hoop stress concentrations at the surface that develop during the anisotropic expansion of crystalline Si. More details on stress evolution and modeling will be presented in section 3 .

Ge reacts via a two-phase mechanism in a similar fashion to $\mathrm{Si}$, but in situ and ex situ experiments have shown that the critical fracture size for Ge pillars $(\sim 1.2 \mu \mathrm{m})$ is much larger than for Si $(\sim 300 \mathrm{~nm})$ during initial lithiation[22, 35, 46, 47]. Note that the critical fracture size for Si pillars $(\sim 300 \mathrm{~nm})$ is larger than for Si particles, as the stress magnitudes in the different structures are expected to be different. The reaction of $\mathrm{Li}^{+}$with $\mathrm{Ge}$ is only slightly anisotropic, and this has been shown to reduce the magnitude of the hoop stress concentrations at the surface during lithiation, leading to greater critical fracture size[46, 47]. Another interesting observation is that delithiation of Ge causes significant porosity to form within nanostructures[26]; such porosity is not usually observed after delithiation of Si. The evolution of porosity during 
delithiation reduces the total volumetric contraction and may be a pathway towards controlling volume changes[46].

Sn is another attractive alloying anode material for Li-ion and Na-ion batteries due to its high lithium storage capacity. In contrast to the brittle semiconductors Si and Ge, Sn is a ductile and malleable metal, and its reaction mechanisms and mechanical degradation have been shown to be different. In situ transmission X-ray microscopy (TXM) studies on the lithiation/delithiation of $\sim 10 \mu$ mdiameter Sn particles have shown a core-shell reaction process and the growth of radial cracks during lithiation[48, 49]. Porosity was also evident after delithiation. More recent in situ TEM studies on (de)lithiation and (de)sodiation of Sn nanoparticles and nanowires have shown complex size-dependent mechanical degradation behaviors, with multiple phase transformations that occur sequentially during reaction[36, 50, 51]. Particles up to a few hundred nm in diameter do not fracture upon lithiation or sodiation, but alkali ion removal causes significant fracture, pulverization, and pore growth within the active material[36, 50]. In addition, fusion of smaller lithiated particles has been observed [36]. Other recent work utilizing in situ synchrotron X-ray tomography hasrevealed real-time changes in the three-dimensional microstructure of active micron-scale $\mathrm{Sn}$ particles in $\mathrm{Li}$ - and Na-ion batteries, as shown in Figure 2e [37, 52]. These investigations have revealed that the first two charge/discharge cycles result in the most mechanical damage to electrode particles, with the material morphology stabilizing after a few cycles [37]. Interestingly, less pulverization has been observed during desodiation of micron-sized Sn particles compared to delithiation, despite greater volume changes during desodiation because of the larger atomic radius of $\mathrm{Na}^{+}[52]$.Together, these studies haveshown that the differing mechanical degradation behaviors in Sn, Ge, and Si depend on the details of the reaction process and stress evolution in each 
material. In addition to studies on these materials, in situ TEM has been used to examine thereaction processes in a variety of other candidate battery materials, revealing disparate mechanical degradation pathways[53-56].
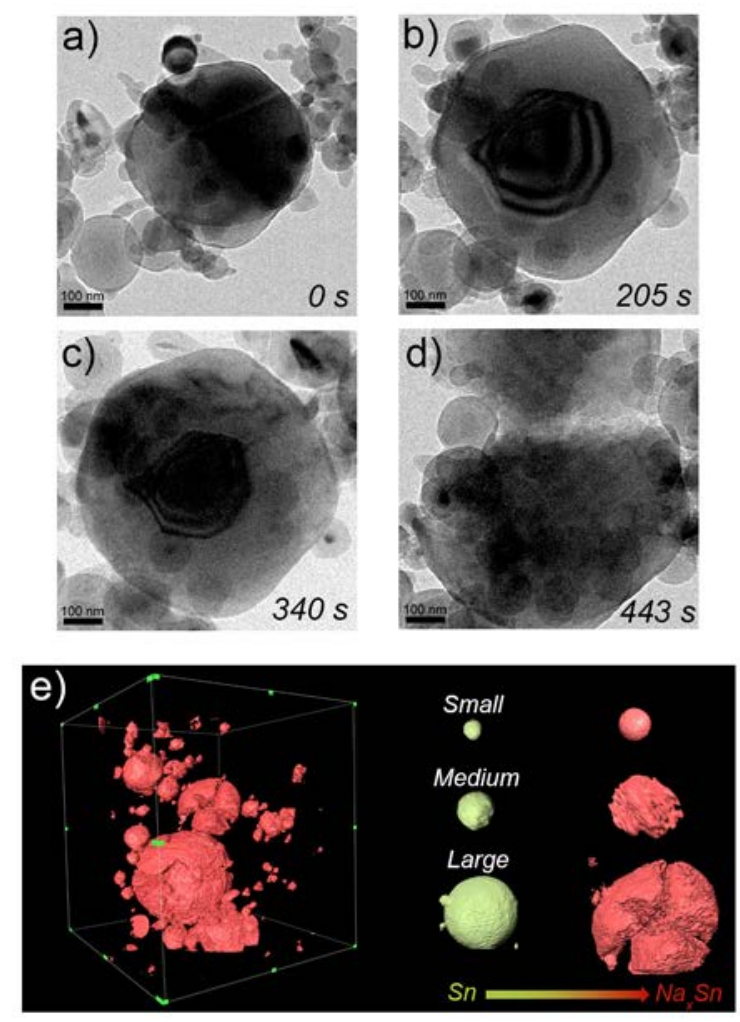

Figure 2. In situ studies of mechanical degradation during reaction of alloying materials. a-d) In situ TEM of the lithiation process of a Si nanoparticle. The particle expands during a two-phase lithiation process and then fractures. The crystalline Si core is visible as the darker interior, while the $\mathrm{Li}_{x} \mathrm{Si}$ shell exhibits lighter contrast.Reproduced with permission [30].Copyright 2012, WILEY-VCH Verlag GmbH \& Co. e)In situ tomographic X-ray images of Sn particles before and aftersodiation. The left frame shows a multi-particle electrode after sodiation (the edge length of the box in this image is $40 \mu \mathrm{m}$ ). The right side shows individual particles of different sizes before and after the sodiation process. The initial diameter of the "small" particle is $0.5 \mu \mathrm{m}$, that of the "medium" particle is $1.6 \mu \mathrm{m}$, and that of the "large" particle is $3.9 \mu \mathrm{m}$. Reproduced with permission[52]. Copyright 2015, Nature Publishing Group.

Beyond thestudies of individual nanoscale structures, mechanical degradation processes in larger particles and thin films have also been investigated. In situ X-ray microscopy techniques have been utilized to study a variety of alloying and conversion materials at the 
microscale within battery electrodes, including $\mathrm{SnO}$, Sb, $\mathrm{NiO}$, and $\mathrm{CuO}[31,33,34,57,58] . \mathrm{In}$ general, such materials with particle size $\sim 5-15 \mu \mathrm{m}$ often undergo acore-shell lithiation process involving the growth of a new lithiated phase, resulting in radial crack formation during the first lithiation. Particles in this size range are presumably larger than the "critical fracture size" for most materials; thus, observations of fracture are prevalent. In addition to reaction processes in micron-scale particles, fracture in thin films of alloying anode materials (especially Si) has been studied. Li et al. have shown that there is a critical thickness below which Si films do not fracture during cycling; this is $\sim 100-200 \mathrm{~nm}$ for Si films on a stainless steel substrate[59]. Furthermore, the fractured area on the substrate was shown to scale with film thickness.Ge films have also shown a similar critical fracture thickness[60]. Patterning of thin films so that lateral dimensions remain below a critical size improves fracture resistance[61]. Finally, recent computational work has revealed cracking mechanisms in thin film Si materials[62].

As noted in this section, progress in in situ experimentation has led to the observation of atomic-to-macroscale reaction processes in a multitude of alloying materials for $\mathrm{Li}$-ion and $\mathrm{Na}$ ionbatteries. Thedetailed knowledge of volume changes, fracture processes, and mechanical degradation has motivated the experimental and computational study of stress evolution, chemomechanical effects, and mechanical properties, which play an outsized role in largevolume-change materials. The remainder of this review is focused on recent work on these topics.

\section{STRESS EVOLUTION WITHIN ACTIVE MATERIALS}

The large volume changes experienced by alloying electrode materials can be reversible during charge/discharge, and such reversible volume changes will not necessarily cause internal 
stresses to develop. However, stress will be generated if the volume changes occur in an inhomogeneous manner or with external mechanical constraints. Inhomogeneous expansion/contraction commonly arises in large-volume-change materials due to the formation of concentration gradients within structures during reaction, which cause gradients in transformation strain and thus large stresses. In addition,mechanical constraints (such as a substrate attached to an active thin film) also cause stress generation. Importantly, the spatiotemporal evolution of stress during large-volume-change transformations dependsstrongly on a variety of factors,includingthe lithiation rate, the geometry of the electrode material (e.g., flat thin films vs. wires/particles), and the reaction mechanism (e.g., two-phase vs. single-phase phase transformations).

In 2010, Sethuraman et al. developed an in situ wafer curvature technique to measure stress evolution in thin film active materials[63]. Since then, a number of studies have been dedicated to measuring stresses in a variety of thin film materials during reaction[60, 64-70]. Figure 3a and b show galvanostatic discharge/charge curves (a) and corresponding measured biaxial stress evolution (b) for a sputtered amorphous Si thin film[63]. Upon lithium insertion, the Si film undergoes elastic loading, followed by yielding at a compressive stress of $\sim 1.5 \mathrm{GPa}$. After yielding, the film undergoes plastic deformation, with flow occurring until the end of lithium insertion (at $\sim 1800 \mathrm{mAh} / \mathrm{g}$ ). Upon lithium removal, the film first undergoes elastic unloading,which is followed by elastic loading and then tensile plastic flow. Other work has shown that the flow stress during lithiation in Si thin films is strongly dependent on the lithiation rate[71], with higher lithiation rates causing larger stresses to exist. This indicates that ratesensitive plasticity occurs in $\mathrm{Li}_{x} \mathrm{Si}$, and it suggests that the lithiation rate will influence the occurrence of fracture. Stress evolution in amorphous Ge films has also been studied [60, 67, 
68]. In general, the flow stresses in $\mathrm{Li}_{x} \mathrm{Ge}$ during lithiation/delithiation have been observed to be less than that for Si thin films[60, 67], as shown in Figure 3c. Rate-sensitive flow stress has also been observed in Ge films[67]. Finally, a recent study has detailed stress evolution in Sn thin films during cycling[69]. As an electrode material, Sn is different than amorphous Si and Ge because Sn undergoes multiple phase transformations during lithiation. These phase transformations influence the biaxial stress during lithiation of Sn films, as seen in Figure 3d. Here, the jumps in voltage represent the formation of new phases, and these events are correlated with jumps in biaxial stress, indicating complex spatiotemporal stress evolution. Taken together, these stress measurements in thin films have revealed the elasto-plastic mechanical response of various important materials, and they have also improved our understanding of the physical mechanisms involved in large-volume-change transformations.
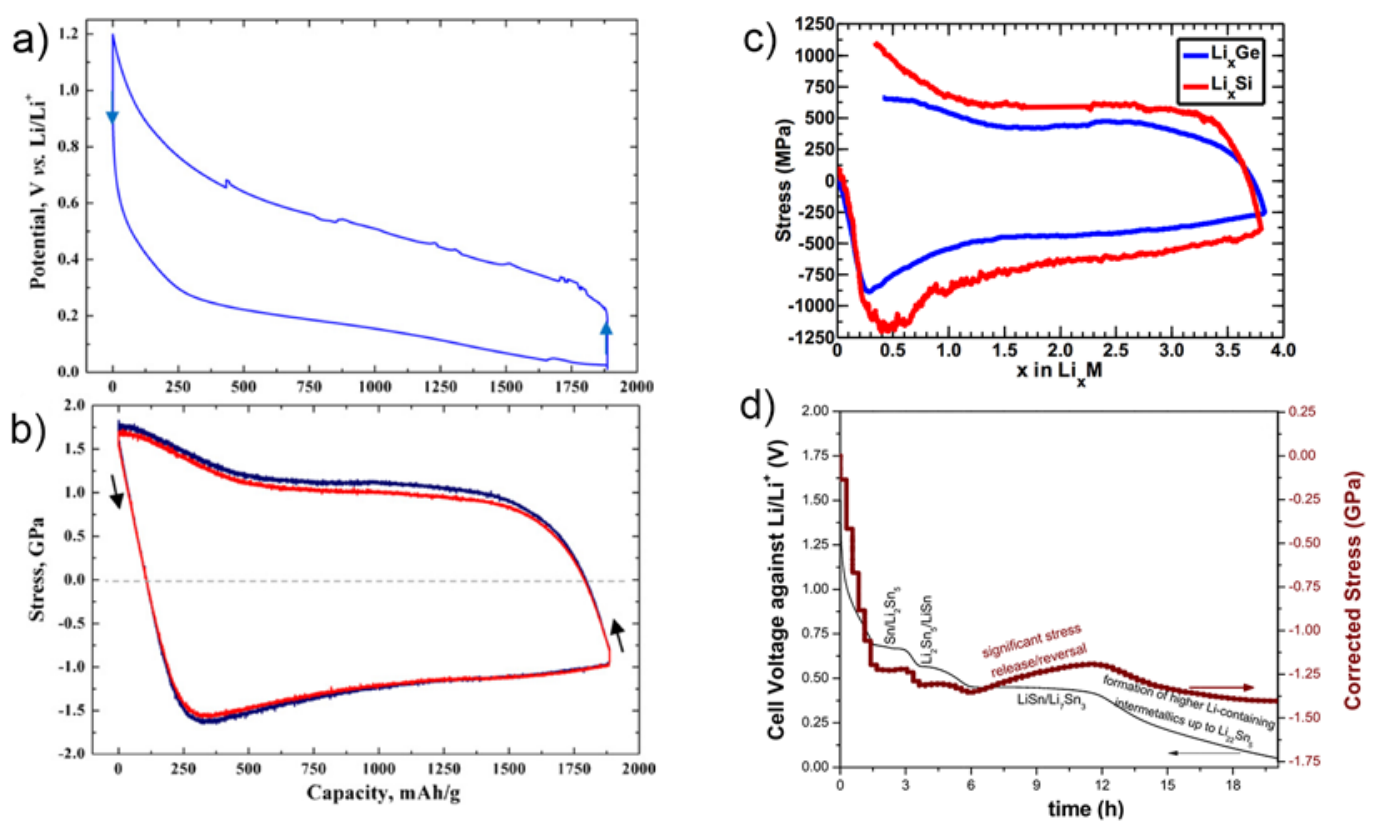

Figure 3.Biaxial stress measured in various thin film materials during lithiation/delithiation. a-b) Galvanostatic potential - capacity plot (a) and measured biaxial stress (b) as a function of extent of lithiation of an amorphous Si thin film electrode cycled at a rate of C/4 (this corresponds to a time of four hours for complete reaction). The extent of lithiation ( $x$-axis) is shown as the lithiation capacity in $\mathrm{mAh} / \mathrm{g}$. Reproduced with permission [63]. Copyright 2010, Elsevier.c) Biaxial stress measured during a lithiation/delithiation cycle for an amorphous Ge thin film and 
an amorphous Si thin film. These electrodes are cycled at a rate of C/16 (16 hours for complete reaction). Reproduced with permission [60]. Copyright 2016, Elsevier.d) Biaxial stress (red) and cell voltage (black) measured during the lithiation of a crystalline Sn thin film. The reaction rate here is C/20. Reproduced with permission [69]. Copyright 2014, Elsevier.

The stress in thin film materials arises primarily because of the substrate constraint during expansion/contraction of the film. The situation is different, however, for the particulate structures that are used in actual battery electrodes: there is no substrate constraint, and the particle surface is not flat. Thus, it would be generally expected that the spatiotemporal stress evolution in particles or wires is different than in thin films; a number of studies have been dedicated to understanding stress evolution in such active particulate structures for large-volumechange materials[72-76]. It should be noted that these studies have been computational or analytical models based on experimental observations of transformations, which is due to the experimental difficulty of measuring real-time stress evolution in particulate structures. Numerical solutions have generally utilized finite element simulations in which stresses are calculated in response to a prescribed transformation strain basedonexperimental observations [73].

Compared to thin films, modeling has shown that both the reaction mechanism and the curvature of the surface affect spatiotemporal stress evolution in particulate structures. In situ TEM experiments have shown that certain materials (such as crystalline $\mathrm{Si}$ ) react via a "twophase” mechanism in which a sharp reaction front separates a highly lithiated phase from the unreacted phase (see Figure 2a-d) [21, 28, 39]. Other materials undergo “single-phase” reaction mechanisms, in which volume expansion is controlled by the diffusion of Li or Na within the active material. Two-phase reactions in a spherical particle involve the movement of a curved reaction front into the particle (Figure 2a-d), while single-phase reactions feature an extended 
radial distribution of $\mathrm{Li}$ [73]. Chemomechanical models have shown that the stress that evolves during lithiation of spherical particles in these two cases is quite different. In particular, lithiated material near the curved reaction front in the two-phase case experiences large compressive stresses, while material near the surface experiences hoop tension (Figure 4a and b) [72, 73].The hoop tension at the surface is a consequence of the curved reaction front, and it can presumably cause fracture, as has been observed in experiments [24]. For the single-phase case, the hoop stress remains compressive near the surface (Figure 4c and d). Other work has shown that for hollow structures (like those increasingly used in electrochemical tests [20, 77]), lithiationinduced stresses are lower during large-volume-change elasto-plastic transformations[78]. Finally, recent studies have investigated the effects of more complex surface curvature and anisotropic expansion behavior on stress evolution [79, 80].
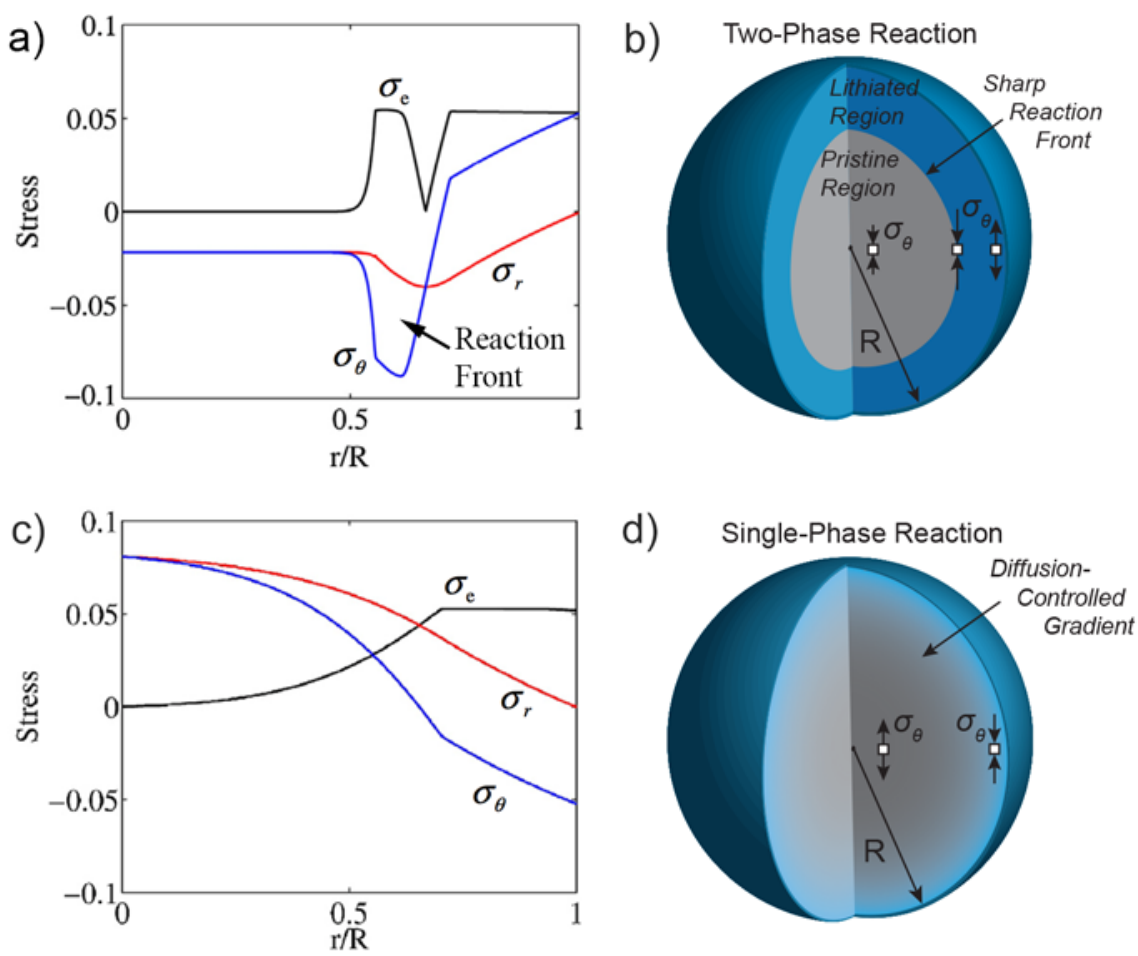

Figure 4.Chemomechanicalmodeling of stress generation in spherical Si particlesin the midst of (a, b) two-phase lithiation and (c, d) single-phase lithiation reactions.The graphs in (a) and(c) show calculated stress profiles, and the schematics in (b) and (d) show the sign of the hoop stress $\sigma_{\theta}$ in different locations. In the schematics, the shade of blue represents the Li concentration 
(darker blue signifies higher concentration).The two-phase reaction in (a) and (b) features a sharp change in Li concentration at the reaction front region, which is represented as an abrupt color change in the schematic in (b). The single-phase reaction in (c) and (d) features a graded lithium concentration, with the highest concentration at the surface of the particle $(r / R=1)$; this is represented as a color gradient in the schematic in (d). The radial distance $r$ on the x-axis of the graphs is normalized by $R$, the total radius of each particle. The stress on the y-axis of the graphs is normalized by Young's modulus. $\sigma_{e}$ is the von Mises effective stress, $\sigma_{r}$ is the radial stress, and $\sigma_{\theta}$ is the hoop stress.Data are reproduced with permission [73]. Copyright 2013, Elsevier.

As evidenced from the studies discussed in this section, inelastic deformation and plasticity play important roles in the stress evolution during reaction of large-volume-change materials. The occurrence of plasticity during these reactions has a number of effects, includinginfluencing the magnitude of the stress that is generated, dissipating energy within the system, and also facilitating morphological changes within active material[81]. Mechanisms of plastic flow are of particular interest in large-volume-change battery materials, as they are presumably quite different than conventional deformation-induced flow in metals. Plastic flow during reaction of amorphous $\mathrm{Li}_{x} \mathrm{Si}$ has been postulated to be influenced by the atomic-scale forming/breaking of Li-Si bonds during reaction[82]. Furthermore, the flow stress is dependent on the reaction rate [71], and it has been predicted to depend on the local hydrostatic stress[83]. Interestingly, other plastic deformation mechanisms have been observed in different materials; for instance, lithiation of $\mathrm{SnO}_{2}$ induces the formation of a dense cloud of dislocations near the reaction front [27]. Such unique plastic deformation mechanisms are not well-understood, and further work in this area may reveal rich and useful insight.

\section{CHEMOMECHANICAL EFFECTS}

The stresses that develop during the reaction of large-volume-change battery materials have been predicted to be large enough to influence the reaction process itself [72]. Such chemomechanical effects can be manifested either as the influence of stress on the 
thermodynamic driving force (i.e., Gibbs free energy) for the electrochemical reaction, or as the influence of stress on ionicdiffusion rates within a material[84-86]. The coupling between biaxial stress and electrochemical potential for $\mathrm{Li}_{x} \mathrm{Si}$ has been measured to be $100 \mathrm{mV} / \mathrm{GPa}$ [87].A number of interesting phenomena attributed to chemomechanical effects have been experimentally observed in large-volume-change systems. In crystalline Si nanowires and nanoparticles, in situ TEM experiments have shown that the lithiation rate decreases significantly with the extent of lithiation [23, 30]. Example data for a nanowire are shown in Figure 5. Analysis of the predicted stresses in both of these cases showed that the increasing magnitude of hydrostatic compressive stress near the reaction front served to diminish either the thermodynamic driving force for the reaction or the rate of diffusion. The increasing stress magnitude with the extent of lithiation is a consequence of the curved reaction front within particles and nanowires, and these observations have ramifications for the maximum attainable lithiation rate in real battery electrodes that contain particles.Other efforts have been focused on attempts to control or alter the lithiation rate in Ge nanowires by applying external bending strains to individual nanowires [88]. These experiments revealed that lithiation proceeded more quickly at the tensile side, whereas lithiation was slower at the compressive side, in agreement with chemomechanical modeling. 

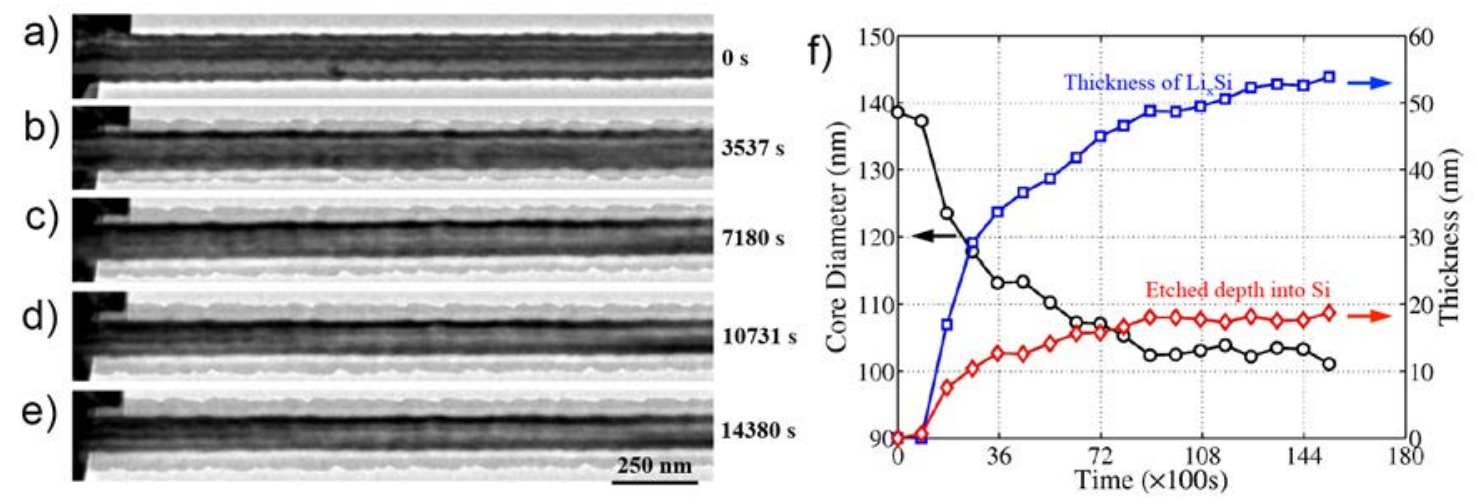

Figure 5.Self-limiting lithiation in a single Si nanowire revealed with in situ TEM.a-e) Images of a Si nanowire during lithiation over the course of 4 hours; the darker core region is crystalline $\mathrm{Si}$, while the lighter shell is lithiated Si. f) Dependence of the core diameter, etched depth of Si, and thickness of $\mathrm{Li}_{x} \mathrm{Si}$ region on time during reaction. Reproduced with permission [23]. Copyright 2013, American Chemical Society.

Additional focus has been directed towards the chemomechanical effects of thin coatings on the surface of nanostructured active materials, as coatings represent a promising route for controlling the surface chemistry and morphological changes of large-volume-change materials.Inert metal coatings on Si nanostructures, such as $\mathrm{Ni}$ and $\mathrm{Cu}$, have been shown to have the ability to "direct" volume expansion by altering the morphological changes, stress evolution, and fracture properties of nanostructures [29, 89].In addition, polymer coatings have been observed to cause the self-delithiation of individual lithiated Si nanoparticles, which has been postulated to be caused by the compressive stress in the nanoparticle interior that is induced by the coating[90].

\section{MECHANICAL PROPERTIES OF ACTIVE MATERIALS}

Predictive computational modeling is a promising route to aid in the accelerated design and development of new electrode materials. Such modeling efforts require a fundamental 
knowledge of the mechanical properties of active electrode materials, including their elastic, plastic, visco-elastoplastic, and fracture properties. However, quantitative characterization of mechanical properties of electrode materials for Li-ion and Na-ion batteries is complicated by a number of factors. First, the mechanical properties of active electrode materials are, in general, a strong function of Li or Na content; this necessitates systematic measurement with varying Li or Na concentrations under well-controlled chemomechanical conditions. Second, the inherent architecture of Li-ion and Na-ion batteries prohibits the use of well-established mechanical testing methods. A Li-ion or Na-ion battery has to operate in a closed inert environment since its electrochemistry is highly sensitive to moisture and oxygen. This constraint adds considerable complexity to experimental design. Third, the electrode materials within a battery have to be connected to a current collector for transferring electrons to the external circuit; the electrical connections usually consist of interspersing the active material within a conductive carbon framework on a metal substrate (Figure 1). For accurate characterization of the electrode material, the mechanical coupling between the electrode and the current collector has to be suppressed or properly accounted for. Finally, recent $a b$ initio studies have revealed that the mechanical properties of active electrode materials may not be solely a function of chemical composition, but may also be affected by dynamic diffusion and reaction processes [91]. This implies that the mechanical properties of lithiated or sodiated electrode materials measured under static conditions could be noticeably different from those obtained during dynamic electrochemical cycling. In recent years, a number of different experimental methodologies have been developed to overcome these difficulties and measure mechanical properties of largevolume-change anode materials. 
The elastic properties of lithiated $\mathrm{Si}$ have been extensively investigated both experimentally and computationally. Sethuraman et al. applied the multi-beam optical stress sensor (MOSS) technique to measure the biaxial modulus of an amorphous Li-Si thin film anode as a function of lithium concentration [92]. Their experiments showed that the biaxial modulus of $\mathrm{Li}_{x} \mathrm{Si}$ decreases from $\sim 70 \mathrm{GPa}$ for $\mathrm{Li}_{0.32} \mathrm{Si}$ to $\sim 35 \mathrm{GPa}$ for $\mathrm{Li}_{3.0} \mathrm{Si}$, largely following a linear rule of mixtures between the properties of pure Si and Li. This result is found to be consistent with the prediction from first principles density functional theory (DFT) calculations[93]. Using in situ high pressure X-ray diffraction, Zeng et al. measured the bulk modulus of polycrystalline $\mathrm{Li}_{3.75} \mathrm{Si}$ [94], a metastable phase that usually forms by crystallization of amorphous $\mathrm{Li}_{3.75} \mathrm{Si}$ upon full lithiation. By fitting the XRD data to the Birch-Murnaghan equation of state, they obtained a bulk modulus of $28.4 \mathrm{GPa}$, which is very close to that which is linearly interpolated from the bulk moduli of pure Si and $\mathrm{Li}$. In addition, this study further showed that the polycrystalline $\mathrm{Li}_{3.75} \mathrm{Si}$ phase remains stable at room temperature under pressures up to $5.8 \mathrm{GPa}$. This result provides a possible explanation for why metastable $c-\mathrm{Li}_{3.75} \mathrm{Si}$ can be formed in $\mathrm{Li}$-ion batteries despite large diffusion-induced stress. 

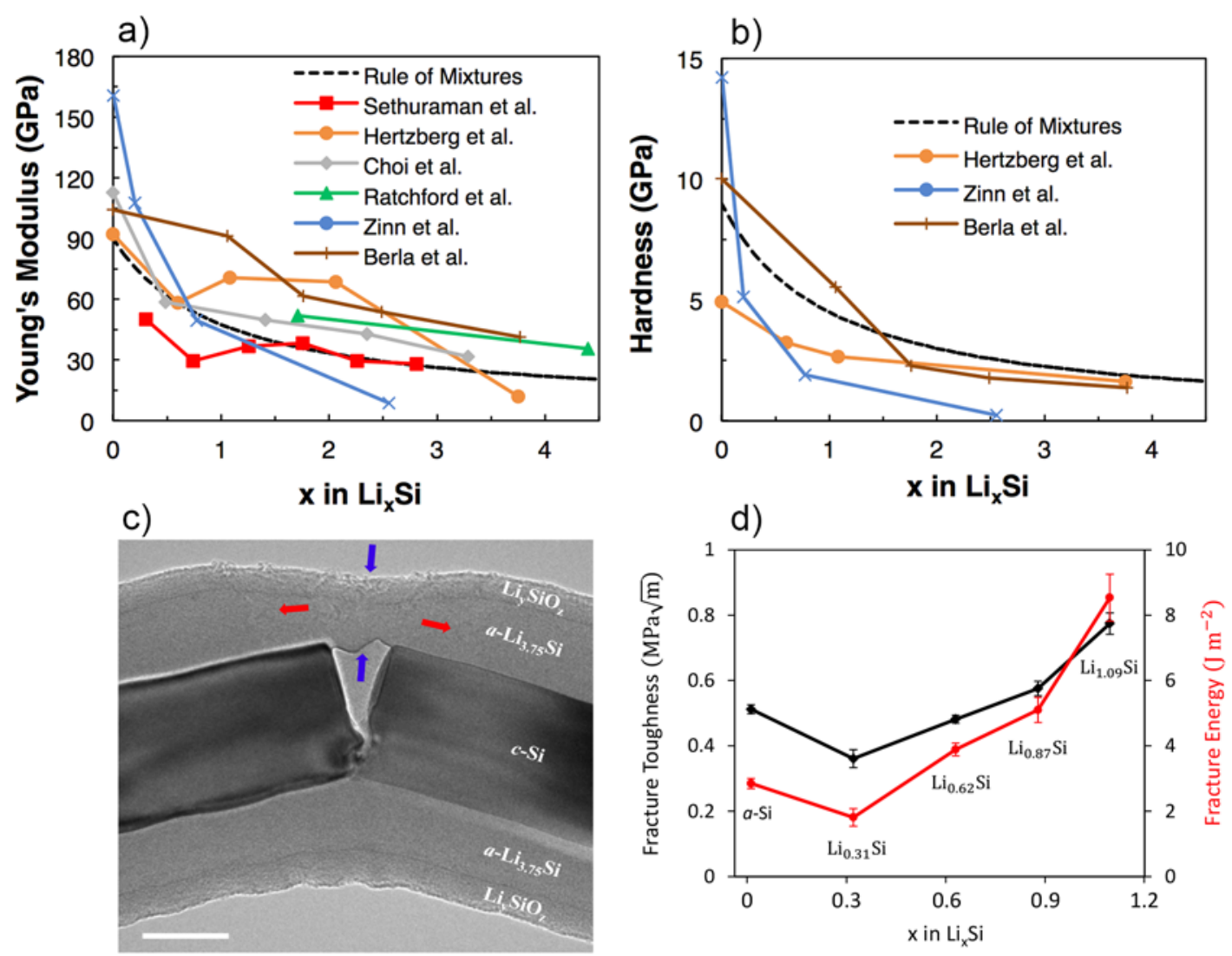

Figure 6. Mechanical properties of lithiated Si. a-b) A compilation of experimental measurements of (a) the Young's modulus and (b) the hardness of $\mathrm{Li}_{x} \mathrm{Si}$ as a function of lithium concentration; these data are taken from a number of different studies. The citations are as follows: Sethuraman [92], Hertzberg [95], Choi [96], Ratchford [97, 98], Zinn [99], Berla [100].c-d) Fracture characteristics of $\mathrm{Li}_{x} \mathrm{Si}$. The TEM image in (c) shows a partially lithiated $\mathrm{Si}$ nanowire subjected to in situ nanomechanical bending test inside a TEM (scale bar, $50 \mathrm{~nm}$ ). The pristine Si core (darker region) is seen to fracture in a brittle manner, while the lithiated Si shell undergoes a large tensile deformation without failure. The plot in (d) shows the fracture toughness and fracture energy of $\mathrm{Li}_{x} \mathrm{Si}$ measured by nanoindentation. A brittle-to-ductile transition of fracture occurs as the lithiation concentration increases. Reproduced with permission [101]. Copyright 2015, Nature Publishing Group.

There is a rich body of work on the characterization of Li-ion battery materials with depth-sensing nanoindentation. Nanoindentation uses a sharp diamond tip to indent the surface of a material at the nanometer scale and yields an indentation load vs. displacement response from which the Young's modulus and hardness of the material can be determined. The smallvolume measurement capability and minimal sample preparation required for nanoindentation 
make it a convenient and effective technique for characterizing lithiated electrode materials. Several groups have measured Young's modulus and the hardness of Li-Si alloys by nanoindentation [95, 97-100]. Some of these studies were performed on amorphous Li-Si thin film electrodes prepared via electrochemical lithiation [95, 99, 100], while others were on polycrystalline Li-Si alloys obtained by high temperature alloying [97, 98]. In order to avoid chemical reaction of lithiated Si with ambient air, great care was taken in these experiments to maintain the samples in mineral oil or inert gas environment during nanoindentation. Data from many of these studies are compiled in Figure 6a-b. As shown in this figure, the reported modulus and hardness data show a relatively large range of values, possibly due to variations in materials synthesis and testing conditions. However, all of these experiments have clearly shown elastic and plastic softening effects caused by lithiation; in other words, both the Young's modulus and hardness decrease as the lithium content increases. Computationally, Qi et al. have performed DFT calculations to investigate the Li-concentration-dependent elastic properties of a large set of electrode materials [102]. This study showed that the Young's modulus of alloy-forming electrode materials (such as $\mathrm{Si}, \beta$-Sn, and $\mathrm{Al}$ ) generally follows a linear rule of mixtures with increasing Li content.

In addition to the elastic properties and hardness of active electrode materials, their stress-strain response is also needed for constitutive modeling of deformation and stress generation. Kushima et al. have performed in situ tensile testing of pristine and lithiated Si nanowires inside a TEM [103]. The average uniaxial tensile strength of tested nanowires was found to decrease from $~ 3.6 \mathrm{GPa}$ in the unlithiated state to $\sim 0.7 \mathrm{GPa}$ in the fully lithiated state. Meanwhile, the fracture strain increased from 2-5\% to 8-16\%. For the lithiated Si nanowires, about $70 \%$ of the tensile deformation was unrecoverable after failure, indicating pronounced 
plasticity in the lithiated Si. Similar in situ tensile testing has been performed by Boles et al. inside a scanning electron microscope (SEM) [104], with the expanded scope of examining the creep behavior of lithiated Si. Creep experiments of fully lithiated Si nanowires were carried out by holding an applied stress and measuring the resultant creep strain. The measured creep strain rate showed a linear relationship with the applied stress when the stress was below the yield strength of the nanowires, which is indicative of quasi-viscous diffusional creep. For stress levels above the yield strength, the creep behavior transitioned to a power-law regime due to the effects of dislocation-mediated viscoplasticity. Furthermore, an inverse exponential relationship with an exponent of 3.3 between the creep strain rate and the nanowire diameter was observed, suggesting that the creep deformation occurred through bulk diffusion.

The time-dependent deformation of lithiated Si has been further investigated by Berla et al. using nanoindentation [100]. By combining quasi-static and continuous stiffness measurement (CSM)-based nanoindentation creep tests, the authors quantitatively studied the creep response of fully lithiated Si over a wide range of strain rates $\left(10^{-5}\right.$ to $\left.10^{-2} \mathrm{~s}^{-1}\right)$. The nanoindentation data analysis revealed a power-law creep behavior with stress exponents of 2030, which are considerably larger than the unity stress exponent reported by the in situ SEM work of Boles et al.[104]. A simple analytical model was developed by assuming that the creep occurs through a thermally activated, shear stress-driven viscous flow mechanism. The model predicted an activation volume that is comparable to the molecular unit volume of fully lithiated Si. This led the authors to suggest that the measured viscoplastic flow occurs by local rearrangement of small atomic clusters rather than individual atoms [100].

The modeling and mitigation of crack formation in active materials also requires the knowledge of Li-concentration-dependent fracture toughness, which is a key property describing 
the ability of a material to resist fracture. In several recent studies, channel cracking of electrochemically cycled or mechanically stretched Si thin-film electrodes was analyzed to infer the fracture energy of lithiated Si at various lithium concentrations $[96,105,106]$. The fracture energy of Li-rich Si was reported to be similar to that of unlithiated Si. This result is in contrast to the more recent work of Wang et al. [101], who detailed the surprising phenomenon of an electrochemically-driven brittle-to-ductile transition during lithiation of Si. In their study, in situ nanomechanical bending tests on partially lithiated Si nanowires were conducted to demonstrate the striking contrast of brittle fracture in pristine Si versus ductile tensile deformation in fully lithiated Si (Figure 6c). Quantitative fracture toughness measurements obtained with nanoindentation showed that lithiated Si exhibited high damage tolerance as the Li:Si molar ratio was increased beyond 1.5 (Figure 6d).To explain the experimentally observed brittle-to-ductile transition, reactive force field (ReaxFF) molecular dynamics (MD) simulations were performed, revealing that the atomic bonding characteristics (i.e., directional covalent Si-Si bonds vs. delocalized metallic Li-Li bonds) were responsible for the lithiation-induced toughening near the crack tip. Notably, the same brittle-to-ductile fracture transition phenomenon has also been recently reported by Ding et al. [107], who used large-scale MD simulations to elucidate a transition in the lithiation-induced fracture mechanism from intrinsic nanoscale cavitation to extensive shear banding ahead of the crack tip. These findings highlight the crucial effects of Li content on the fracture properties of lithiated Si, and they have profound implications for the design of fracture- and damage-tolerant electrodes.

Using the aforementioned nanoindentation method, Wang et al. have also investigated the fracture characteristics of lithiated Ge as a viable high-capacity anode material [108]. Compared to $\mathrm{Si}$, Ge was found to have slightly lower fracture energy in the unlithiated state. 
However, the fracture energy of Ge increases rapidly upon lithiation and far surpasses that of lithiated $\mathrm{Si}$ at moderate to high lithium concentrations. This finding provides a compelling explanation for the superior mechanical robustness of Ge nanoparticles and pillars observed in previous in situ and ex situ Ge lithiation experiments [22, 47], and it confirms the substantial potential of Ge for use in durable high-rate lithium-ion battery electrodes.

\section{INTERFACES AND MESOSCALE PHENOMENA}

The majority of this paper has been focused on the mechanical aspects of large-volumechange reactions in individual structures, such as particles or thin films. However, as shown in

Figure 1, conventional battery electrodes are actually composite architectures made up of a combination of particulate active material, conductive additive, and polymeric binder. The structure of the electrode is designed to maintain both electrical and ionic transport pathways within the architecture throughout the reaction-induced transformation processes.Thus, mesoscale effects are important to manage within electrode architectures; such effects includethe chemomechanical interactions among the various electrode components and the mechanical stability of interfaces[109]. This is especially important when considering large-volume-change active materials, as mechanical interactions among contacting particles, as well as overall volume changes of the electrode, can influence connectivity and thereforeelectrochemical behavior.Understanding mesoscale and interfacial behaviors provides a much-needed link between processes in individual particles and electrochemical performance. In this section, recent work on mesoscale and interfacial mechanics of large-volume-change battery materials is discussed. 
A number of studies have focused on the interfacial strength and adhesionbetween largevolume-change materials and metal current collectors, such as $\mathrm{Cu}$. This is important both for conventional electrode architectures, as well as for thin-film batteries in which the continued adhesion of the thin-film active material during cycling is necessary.In situ X-ray tomography has revealed real-time delamination processes between $\mathrm{Si}$ thin films and $\mathrm{Cu}$ current collectors during reaction[32].Such results can be further understood by considering recent experimental and theoretical work showing the enrichment of $\mathrm{Li}$ at $\mathrm{Si}-\mathrm{Cu}$ interfaces during lithiation of Si thin films on $\mathrm{Cu}$ current collectors $[110,111]$. The high Li concentration alters the mechanical properties of this interface, decreasing both the adhesion strength and sliding resistance between the layers[110]. Decreased sliding resistance could be beneficial, since this would serve to diminish the stress within the active material and may reduce the extent of delamination[111]. However, control of atomic bonding at the interface is crucial for reduced sliding resistance; Si$\mathrm{Cu}$ and $\mathrm{Li}-\mathrm{Cu}$ bonds have been found to be ideal for promoting interfacial sliding, while the formation of $\mathrm{LiSi}_{3}$ compounds at the interface lock the layers together[111]. The importance of controlling interfacial adhesion has been further illustrated in a recent report in which a single layer of graphene was used to alter the mechanics and chemistry of the metal-Si interface; the graphene interlayer greatly improved the capacity retention with cycling of a Si nanowire electrode[112].

Another important area of study is how interactions between particles or nanostructures within a battery electrode influence the stress states within particles. This topic has recently been investigated with a focus on $\mathrm{Si}$ nanostructures during lithiation-induced expansion. An experimental study by Lee et al. demonstrated that interactions between expanding $\mathrm{Si}$ nanostructures altered the volume change behavior and also improved fracture resistance during 
the first lithiation by reducing the influence of anisotropic expansion [113]. Subsequent modeling has shown that the mechanical stress generated due to impinging Si nanostructures during lithiation-induced expansion can significantly alter the stress state and the spatial Li concentration through chemomechanical effects [114]. Such results indicate that particleparticleinteractions within electrode architectures must be carefully engineered, as they can affectinternal stress evolutionin particles.

The largest length scale of importance in Li-ion and Na-ion batteries is that of the entire composite electrode. Volumetric changes and stress/strain evolution at the electrode level aredetermined by processes at shorter length scales, including transformations in individual active particles and interactions between particles.Initial work on understanding electrode-level mechanics has focused on electrodes containing conventional intercalationmaterials, such as graphite and $\mathrm{LiCoO}_{2}$ [115-117]. Recently, a number of authors have studied large-volume-change materials, which feature more dramatic electrode-level changes $[118,119]$. Sethuraman et al. have measured the stress within composite electrode structures containing Si active material during cycling, and they have shown that the choice of polymeric binder significantly influences the magnitude of stress that develops (Figure 7)[120]. Binders that form stronger hydrogen bonds with Si particles (such as carboxymethyl cellulose, CMC) result in larger stress values than binders that interact via weaker van der Waals forces (such as polyvinylidene fluoride, PVDF). These results further show that mesoscale interactions must be controlled for tailored mechanical response in electrode architectures. 

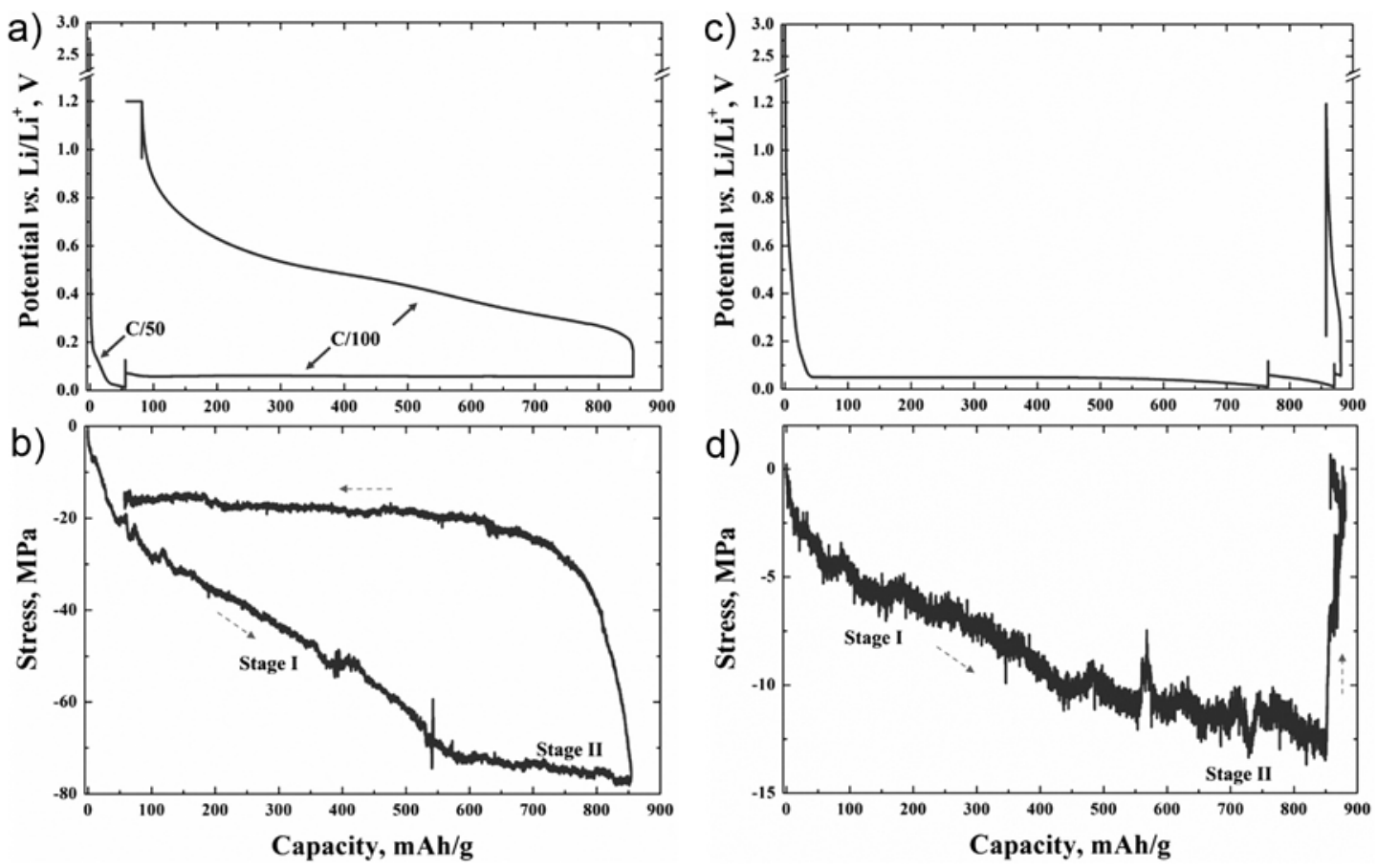

Figure 7. In situ stress measurement during the first lithiation/delithiation cycle of particulate Si electrodes. a-b) Potential-capacity plot (a) and corresponding stress within the electrode (b) for a Si electrode made with CMC binder. c-d) Potential-capacity plot (c) and corresponding stress within the electrode (d) for a Si electrode made with PVDF binder. Reproduced with permission[120]. Copyright 2013, The Electrochemical Society.

In addition to interface adhesion and mesoscale effects, the mechanical aspects of solidelectrolyte interphase (SEI) growth on large-volume-change materials have also begun to receive attention. As discussed previously, the SEI is a film that forms on the surface of anode materials as a result of the reductive decomposition of the electrolyte. It is made up of a mixture of inorganic and organic components. SEI films are electronically insulating, which can potentially cause electronic isolation of active materials within an electrode; furthermore, SEI growth irreversibly consumes $\mathrm{Li}^{+}$and thus decreases the Coulombic efficiency of battery cycling. In conventional anode materials (i.e.,graphite), the growth of the SEI film is selflimiting, but in large-volume-change materials, cyclical volume changes can cause fracture and regrowth of thick SEI films on newly exposed anode surface. To understand the growth and 
fracture of SEI films, it is necessary to measure their mechanical properties. A few studies have used scanning probe techniques to determine the elastic modulus of SEI films on Si electrodes, with reported elastic modulus values varyingbetween a few MPa and a few GPa[121, 122].Recent experiments by Tokranov et al. have shown that the mechanical stiffness of the SEI layer depends on the electrochemical potential at which it is formed[123]. The differing mechanical properties are related to changes in the composition of the film. Furthermore, the mechanical stiffness and ionic conductivity were observed to change during cycling[123]. These results point to the complexity of SEI films, suggesting that more understanding is necessary to control SEI growth during battery operation.

This section has focused on the importance of interfaces and mesoscale effects in influencing the mechanical behaviors of large-volume-change battery electrodes. Although an increasing amount of work has been dedicated to this area in recent years, it remains an active research direction with much remaining work to be done.

\section{CONCLUSIONS}

Recent years have seen enormous research effort dedicated to developing high-capacity, large-volume-change anode materials for Li-ion and Na-ion batteries. Detailed experimental analysis of reaction-induced transformations in these materials has revealed the importance of mechanics in controlling volume changes and mechanical degradation, assignificant reactioninduced stresses and plastic deformation processes are typical. This review has focused on recent studies dedicated to understanding the mechanics-related aspects of these transformations, including mechanical properties of active materials, reaction- and diffusion-induced stress evolution, and chemomechanical effects on reaction processes.In many cases, close coupling 
between in situ experiments to probe transformation processesand modeling to understand underlying mechanisms has provided deep insight beyond what each approach individually can attain. These studies have been necessary for engineering the next generation of damage-tolerant, long cycle life battery anodes.

There is still much to be learned about the mechano-electro-chemical processes in largevolume-change materials and electrodes. In particular, our understanding of the mechanical properties and deformation of SEI layers on large-volume-change materials is still limited. This is complicated by the complex chemical nature of SEI, which can change based on the electrolyte used or the voltage schedule employed. Such understanding will be key for minimizing the formation of SEI on large-volume-change materials. In addition to studying the SEI, another important area is the measurement of stress and strain in individual active structures other than thin films. Most stress measurements of large-volume-change materials have been made on thin film structures using techniques such as the multi-beam optical stress sensor method[63]. Very recent work has utilized Raman spectroscopy to detect stress evolution in Si particles during lithiation[124]. While modeling studies have predicted stresses in particles of different shapes and sizes, more detailed experiments revealing spatiotemporal stress evolution in realistic particleswould also be useful. Understanding and quantifying such stress evolution will enable predictive chemomechanical modeling in large-volume-change materials, essential for rational development of durable high-capacity rechargeable batteries. 


\section{ACKNOWLEDGMENTS}

MTM acknowledges support from Georgia Tech. SX acknowledges support from the National

Science Foundation (CMMI-1300458). TZ acknowledges support from the National Science Foundation (CMMI-1100205 and DMR-1410936). 


\section{REFERENCES}

[1] B. Dunn, H. Kamath, J.-M. Tarascon, Electrical energy storage for the grid: A battery of choices, Science 334 (2011) 928-35.

[2] M. S. Whittingham, History, evolution, and future status of energy storage, Proc. IEEE 100 (2012) 1518-34.

[3] X. Su, Q. Wu, J. Li, X. Xiao, A. Lott, W. Lu, B. W. Sheldon, J. Wu, Silicon-based nanomaterials for lithium-ion batteries: A review, Adv. Energy Mater. 4 (2014) 1300882.

[4] N. Nitta, G. Yushin, High-capacity anode materials for lithium-ion batteries: Choice of elements and structures for active particles, Particle \& Particle Systems Characterization 31 (2014) 317-36.

[5] M. T. McDowell, S. W. Lee, W. D. Nix, Y. Cui, 25th anniversary article: Understanding the lithiation of silicon and other alloying anodes for lithium-ion batteries, Adv. Mater. 25 (2013) 4966-85.

[6] R. Marom, S. F. Amalraj, N. Leifer, D. Jacob, D. Aurbach, A review of advanced and practical lithium battery materials, J. Mater. Chem. 21 (2011) 9938.

[7] W.-J. Zhang, A review of the electrochemical performance of alloy anodes for lithium-ion batteries, J. Power Sources 196 (2011) 13-24.

[8] W.-J. Zhang, Lithium insertion/extraction mechanism in alloy anodes for lithium-ion batteries, J. Power Sources 196 (2011) 877-85.

[9] U. Kasavajjula, C. Wang, A. J. Appleby, Nano- and bulk-silicon-based insertion anodes for lithium-ion secondary cells, J. Power Sources 163 (2007) 1003-39.

[10] S. S. Zhang, Liquid electrolyte lithium/sulfur battery: Fundamental chemistry, problems, and solutions, J. Power Sources 231 (2013) 153-62.

[11] G. Girishkumar, B. McCloskey, A. C. Luntz, S. Swanson, W. Wilcke, Lithium-air battery: Promise and challenges, J. Phys. Chem. Lett. 1 (2010) 2193-203.

[12] M. Skyllas-Kazacos, M. H. Chakrabarti, S. A. Hajimolana, F. S. Mjalli, M. Saleem, Progress in flow battery research and development, J. Electrochem. Soc. 158 (2011) R55.

[13] S.-W. Kim, D.-H. Seo, X. Ma, G. Ceder, K. Kang, Electrode materials for rechargeable sodium-ion batteries: Potential alternatives to current lithium-ion batteries, Adv. Energy Mater. 2 (2012) 710-21. 
[14] R. A. Huggins. Advanced batteries: Materials science aspects. New York, NY: Springer Science+Business Media; 2009.

[15] N. Nitta, F. Wu, J. T. Lee, G. Yushin, Li-ion battery materials: Present and future, Mater. Today 18 (2015) 252-64.

[16] M. N. Obrovac, V. L. Chevrier, Alloy negative electrodes for Li-ion batteries, Chem. Rev. 114 (2014) 11444-502.

[17] J. Cabana, L. Monconduit, D. Larcher, M. R. Palacin, Beyond intercalation-based Li-ion batteries: The state of the art and challenges of electrode materials reacting through conversion reactions, Adv. Mater. 22 (2010) E170-92.

[18] A. Mukhopadhyay, B. W. Sheldon, Deformation and stress in electrode materials for Li-ion batteries, Prog. Mater. Science 63 (2014) 58-116.

[19] N. Liu, Z. Lu, J. Zhao, M. T. McDowell, H. W. Lee, W. Zhao, Y. Cui, A pomegranateinspired nanoscale design for large-volume-change lithium battery anodes, Nat. Nanotechnol. 9 (2014) 187-92.

[20] Y. Yao, M. T. McDowell, I. Ryu, H. Wu, N. Liu, L. Hu, W. D. Nix, Y. Cui, Interconnected silicon hollow nanospheres for lithium-ion battery anodes with long cycle life, Nano Lett. 11 (2011) 2949-54.

[21] J. W. Wang, Y. He, F. Fan, X. H. Liu, S. Xia, Y. Liu, C. T. Harris, H. Li, J. Y. Huang, S. X. Mao, T. Zhu, Two-phase electrochemical lithiation in amorphous silicon, Nano Lett. 13 (2013) 709-15.

[22] W. Liang, H. Yang, F. Fan, Y. Liu, X. H. Liu, J. Y. Huang, T. Zhu, S. Zhang, Tough germanium nanoparticles under electrochemical cycling, ACS Nano 7 (2013) 3427-33.

[23] X. H. Liu, F. Fan, H. Yang, S. Zhang, J. Y. Huang, T. Zhu, Self-limiting lithiation in silicon nanowires, ACS Nano 7 (2013) 1495-503.

[24] X. H. Liu, L. Zhong, S. Huang, S. X. Mao, T. Zhu, J. Y. Huang, Size-dependent fracture of silicon nanoparticles during lithiation, ACS Nano 6 (2012) 1522-31.

[25] X. H. Liu, H. Zheng, L. Zhong, S. Huang, K. Karki, L. Q. Zhang, Y. Liu, A. Kushima, W. T. Liang, J. W. Wang, J. H. Cho, E. Epstein, S. A. Dayeh, S. T. Picraux, T. Zhu, J. Li, J. P. Sullivan, J. Cumings, C. Wang, S. X. Mao, Z. Z. Ye, S. Zhang, J. Y. Huang, Anisotropic swelling and fracture of silicon nanowires during lithiation, Nano Lett. 11 (2011) 3312-8. 
[26] X. H. Liu, S. Huang, S. T. Picraux, J. Li, T. Zhu, J. Y. Huang, Reversible nanopore formation in Ge nanowires during lithiation-delithiation cycling: An in situ transmission electron microscopy study, Nano Lett. 11 (2011) 3991-7.

[27] J. Y. Huang, L. Zhong, C. M. Wang, J. P. Sullivan, W. Xu, L. Q. Zhang, S. X. Mao, N. S. Hudak, X. H. Liu, A. Subramanian, H. Fan, L. Qi, A. Kushima, J. Li, In situ observation of the electrochemical lithiation of a single $\mathrm{SnO}_{2}$ nanowire electrode, Science 330 (2010) 1515-20.

[28] M. T. McDowell, S. W. Lee, J. T. Harris, B. A. Korgel, C. Wang, W. D. Nix, Y. Cui, In situ TEM of two-phase lithiation of amorphous silicon nanospheres, Nano Lett. 13 (2013) 75864.

[29] M. T. McDowell, S. Woo Lee, C. Wang, Y. Cui, The effect of metallic coatings and crystallinity on the volume expansion of silicon during electrochemical lithiation/delithiation, Nano Energy 1 (2012) 401-10.

[30] M. T. McDowell, I. Ryu, S. W. Lee, C. Wang, W. D. Nix, Y. Cui, Studying the kinetics of crystalline silicon nanoparticle lithiation with in situ transmission electron microscopy, Adv. Mater. 24 (2012) 6034-41.

[31] C. Villevieille, M. Ebner, J. L. Gomez-Camer, F. Marone, P. Novak, V. Wood, Influence of conversion material morphology on electrochemistry studied with operando x-ray tomography and diffraction, Adv. Mater. 27 (2015) 1676-81.

[32] F. Tariq, V. Yufit, D. S. Eastwood, Y. Merla, M. Biton, B. Wu, Z. Chen, K. Freedman, G. Offer, E. Peled, P. D. Lee, D. Golodnitsky, N. Brandon, In-operando x-ray tomography study of lithiation induced delamination of Si based anodes for lithium-ion batteries, ECS Electrochem. Lett. 3 (2014) A76-A8.

[33] M. Ebner, F. Marone, M. Stampanoni, V. Wood, Visualization and quantification of electrochemical and mechanical degradation in Li ion batteries, Science 342 (2013) 71620.

[34] J. Nelson Weker, M. F. Toney, Emerging in situ and operando nanoscale x-ray imaging techniques for energy storage materials, Adv. Func. Mater. 25 (2015) 1622-37.

[35] J. N. Weker, N. Liu, S. Misra, J. C. Andrews, Y. Cui, M. F. Toney, In situ nanotomography and operando transmission x-ray microscopy of micron-sized Ge particles, Energy Environ. Science 7 (2014) 2771. 
[36] J. Wang, F. Fan, Y. Liu, K. L. Jungjohann, S. W. Lee, S. X. Mao, X. Liu, T. Zhu, Structural evolution and pulverization of tin nanoparticles during lithiation-delithiation cycling, J. Electrochem. Soc. 161 (2014) F3019-F24.

[37] J. Wang, Y. C. Chen-Wiegart, J. Wang, In situ three-dimensional synchrotron X-ray nanotomography of the (de)lithiation processes in tin anodes, Angew. Chem. Int. Ed. Engl. 53 (2014) 4460-4.

[38] Z. Li, X. Tan, P. Li, P. Kalisvaart, M. T. Janish, W. M. Mook, E. J. Luber, K. L. Jungjohann, C. B. Carter, D. Mitlin, Coupling in situ TEM and ex situ analysis to understand heterogeneous sodiation of antimony, Nano Lett. 15 (2015) 6339-48.

[39] X. H. Liu, J. W. Wang, S. Huang, F. Fan, X. Huang, Y. Liu, S. Krylyuk, J. Yoo, S. A. Dayeh, A. V. Davydov, S. X. Mao, S. T. Picraux, S. Zhang, J. Li, T. Zhu, J. Y. Huang, In situ atomic-scale imaging of electrochemical lithiation in silicon, Nat. Nanotechnol. 7 (2012) 749-56.

[40] S. W. Lee, M. T. McDowell, L. A. Berla, W. D. Nix, Y. Cui, Fracture of crystalline silicon nanopillars during electrochemical lithium insertion, Proc. Natl. Acad. Sci. USA 109 (2012) 4080-5.

[41] I. Ryu, J. W. Choi, Y. Cui, W. D. Nix, Size-dependent fracture of Si nanowire battery anodes, J. Mech. Phys. Solids 59 (2011) 1717-30.

[42] S. W. Lee, M. T. McDowell, J. W. Choi, Y. Cui, Anomalous shape changes of silicon nanopillars by electrochemical lithiation, Nano Lett. 11 (2011) 3034-9.

[43] J. L. Goldman, B. R. Long, A. A. Gewirth, R. G. Nuzzo, Strain anisotropies and selflimiting capacities in single-crystalline 3D silicon microstructures: Models for high energy density lithium-ion battery anodes, Adv. Func. Mater. 21 (2011) 2412-22.

[44] H. Yang, S. Huang, X. Huang, F. Fan, W. Liang, X. H. Liu, L. Q. Chen, J. Y. Huang, J. Li, T. Zhu, S. Zhang, Orientation-dependent interfacial mobility governs the anisotropic swelling in lithiated silicon nanowires, Nano Lett. 12 (2012) 1953-8.

[45] L. A. Berla, S. W. Lee, I. Ryu, Y. Cui, W. D. Nix, Robustness of amorphous silicon during the initial lithiation/delithiation cycle, J. Power Sources 258 (2014) 253-9.

[46] Y. Liu, S. Zhang, T. Zhu, Germanium-based electrode materials for lithium-ion batteries, ChemElectroChem 1 (2014) 706-13. 
[47] S. W. Lee, I. Ryu, W. D. Nix, Y. Cui, Fracture of crystalline germanium during electrochemical lithium insertion, Extreme Mech. Lett. 2 (2015) 15-9.

[48] S.-C. Chao, Y.-F. Song, C.-C. Wang, H.-S. Sheu, H.-C. Wu, N.-L. Wu, Study on microstructural deformation of working $\mathrm{Sn}$ and $\mathrm{SnSb}$ anode particles for Li-ion batteries by in situ transmission x-ray microscopy, J. Phys. Chem. C 115 (2011) 22040-7.

[49] S.-C. Chao, Y.-C. Yen, Y.-F. Song, Y.-M. Chen, H.-C. Wu, N.-L. Wu, A study on the interior microstructures of working Sn particle electrode of Li-ion batteries by in situ x-ray transmission microscopy, Electrochem. Commun. 12 (2010) 234-7.

[50] Q. Li, P. Wang, Q. Feng, M. Mao, J. Liu, S. X. Mao, H. Wang, In situ TEM on the reversibility of nanosized Sn anodes during the electrochemical reaction, Chem. Mater. 26 (2014) 4102-8.

[51] J. W. Wang, X. H. Liu, S. X. Mao, J. Y. Huang, Microstructural evolution of tin nanoparticles during in situ sodium insertion and extraction, Nano Lett. 12 (2012) 5897902.

[52] J. Wang, C. Eng, Y. C. Chen-Wiegart, J. Wang, Probing three-dimensional sodiationdesodiation equilibrium in sodium-ion batteries by in situ hard x-ray nanotomography, Nat. Commun. 6 (2015) 7496.

[53] Y. Liu, N. S. Hudak, D. L. Huber, S. J. Limmer, J. P. Sullivan, J. Y. Huang, In situ transmission electron microscopy observation of pulverization of aluminum nanowires and evolution of the thin surface $\mathrm{Al}_{2} \mathrm{O}_{3}$ layers during lithiation-delithiation cycles, Nano Lett. 11 (2011) 4188-94.

[54] L. Q. Zhang, X. H. Liu, Y. Liu, S. Huang, T. Zhu, L. Gui, S. X. Mao, Z. Z. Ye, C. M. Wang, J. P. Sullivan, J. Y. Huang, Controlling the lithiation-induced strain and charging rate in nanowire electrodes by coating, ACS Nano 5 (2011) 4800-9.

[55] A. Kushima, X. H. Liu, G. Zhu, Z. L. Wang, J. Y. Huang, J. Li, Leapfrog cracking and nanoamorphization of $\mathrm{ZnO}$ nanowires during in situ electrochemical lithiation, Nano Lett. 11 (2011) 4535-41.

[56] M. T. McDowell, Z. Lu, K. J. Koski, J. H. Yu, G. Zheng, Y. Cui, In situ observation of divergent phase transformations in individual sulfide nanocrystals, Nano Lett. 15 (2015) 1264-71. 
[57] J. Wang, Y. C. Chen-Wiegart, J. Wang, In situ chemical mapping of a lithium-ion battery using full-field hard x-ray spectroscopic imaging, Chem. Commun. 49 (2013) 6480-2.

[58] J. Gonzalez, K. Sun, M. Huang, J. Lambros, S. Dillon, I. Chasiotis, Three dimensional studies of particle failure in silicon based composite electrodes for lithium ion batteries, J. Power Sources 269 (2014) 334-43.

[59] J. Li, A. K. Dozier, Y. Li, F. Yang, Y.-T. Cheng, Crack pattern formation in thin film lithium-ion battery electrodes, J. Electrochem. Soc. 158 (2011) A689.

[60] M. Pharr, Y. S. Choi, D. Lee, K. H. Oh, J. J. Vlassak, Measurements of stress and fracture in germanium electrodes of lithium-ion batteries during electrochemical lithiation and delithiation, J. Power Sources 304 (2016) 164-9.

[61] X. Xiao, P. Liu, M. W. Verbrugge, H. Haftbaradaran, H. Gao, Improved cycling stability of silicon thin film electrodes through patterning for high energy density lithium batteries, J. Power Sources 196 (2011) 1409-16.

[62] H. B. Chew, B. Hou, X. Wang, S. Xia, Cracking mechanisms in lithiated silicon thin film electrodes, Int. J. Solids Struct. 51 (2014) 4176-87.

[63] V. A. Sethuraman, M. J. Chon, M. Shimshak, V. Srinivasan, P. R. Guduru, In situ measurements of stress evolution in silicon thin films during electrochemical lithiation and delithiation, J. Power Sources 195 (2010) 5062-6.

[64] G. Bucci, S. P. V. Nadimpalli, V. A. Sethuraman, A. F. Bower, P. R. Guduru, Measurement and modeling of the mechanical and electrochemical response of amorphous Si thin film electrodes during cyclic lithiation, J. Mech. Phys. Solids 62 (2014) 276-94.

[65] S. P. V. Nadimpalli, V. A. Sethuraman, G. Bucci, V. Srinivasan, A. F. Bower, P. R. Guduru, On plastic deformation and fracture in $\mathrm{Si}$ films during electrochemical lithiation/delithiation cycling, J. Electrochem. Soc. 160 (2013) A1885-A93.

[66] M. J. Chon, V. A. Sethuraman, A. McCormick, V. Srinivasan, P. R. Guduru, Real-time measurement of stress and damage evolution during initial lithiation of crystalline silicon, Phys. Rev. Lett. 107 (2011) 045503.

[67] S. P. Nadimpalli, R. Tripuraneni, V. A. Sethuraman, Real-time stress measurements in germanium thin film electrodes during electrochemical lithiation/delithiation cycling, J. Electrochem. Soc. 162 (2015) A2840-A6. 
[68] A. Al-Obeidi, D. Kramer, C. V. Thompson, R. Mönig, Mechanical stresses and morphology evolution in germanium thin film electrodes during lithiation and delithiation, J. Power Sources 297 (2015) 472-80.

[69] A. Mukhopadhyay, R. Kali, S. Badjate, A. Tokranov, B. W. Sheldon, Plastic deformation associated with phase transformations during lithiation/delithiation of Sn, ScriptaMater. 92 (2014) 47-50.

[70] S. K. Soni, B. W. Sheldon, X. Xiao, A. Tokranov, Thickness effects on the lithiation of amorphous silicon thin films, Scripta Mater. 64 (2011) 307-10.

[71] M. Pharr, Z. Suo, J. J. Vlassak, Variation of stress with charging rate due to strain-rate sensitivity of silicon electrodes of Li-ion batteries, J. Power Sources 270 (2014) 569-75.

[72] K. Zhao, M. Pharr, Q. Wan, W. L. Wang, E. Kaxiras, J. J. Vlassak, Z. Suo, Concurrent reaction and plasticity during initial lithiation of crystalline silicon in lithium-ion batteries, J. Electrochem. Soc. 159 (2012) A238-A43.

[73] S. Huang, F. Fan, J. Li, S. Zhang, T. Zhu, Stress generation during lithiation of highcapacity electrode particles in lithium ion batteries, Acta Mater. 61 (2013) 4354-64.

[74] A. F. Bower, P. R. Guduru, E. Chason, Analytical solutions for composition and stress in spherical elastic-plastic lithium-ion electrode particles containing a propagating phase boundary, Int. J. Solids Struct. 69-70 (2015) 328-42.

[75] L. Chen, F. Fan, L. Hong, J. Chen, Y. Z. Ji, S. L. Zhang, T. Zhu, L. Q. Chen, A phase-field model coupled with large elasto-plastic deformation: Application to lithiated silicon electrodes, J. Electrochem. Soc. 161 (2014) F3164-F72.

[76] H. Yang, F. Fan, W. Liang, X. Guo, T. Zhu, S. Zhang, A chemo-mechanical model of lithiation in silicon, J. Mech. Phys. Solids 70 (2014) 349-61.

[77] H. Wu, G. Chan, J. W. Choi, I. Ryu, Y. Yao, M. T. McDowell, S. W. Lee, A. Jackson, Y. Yang, L. B. Hu, Y. Cui, Stable cycling of double-walled silicon nanotube battery anodes through solid-electrolyte interphase control, Nat. Nanotechnol. 7 (2012) 310-5.

[78] Z. Jia, T. Li, Stress-modulated driving force for lithiation reaction in hollow nano-anodes, J. Power Sources 275 (2015) 866-76.

[79] Y. Lu, Y. Ni, Effects of particle shape and concurrent plasticity on stress generation during lithiation in particulate Li-ion battery electrodes, Mech. Mater.91 (2015) 372-81. 
[80] I. Ryu, S. W. Lee, H. Gao, Y. Cui, W. D. Nix, Microscopic model for fracture of crystalline Si nanopillars during lithiation, J. Power Sources 255 (2014) 274-82.

[81] C. V. Di Leo, E. Rejovitzky, L. Anand, Diffusion-deformation theory for amorphous silicon anodes: The role of plastic deformation on electrochemical performance, Int. J. Solids Struct. 67-68 (2015) 283-96.

[82] K. Zhao, G. A. Tritsaris, M. Pharr, W. L. Wang, O. Okeke, Z. Suo, J. J. Vlassak, E. Kaxiras, Reactive flow in silicon electrodes assisted by the insertion of lithium, Nano Lett. 12 (2012) 4397-403.

[83] K.-J. Zhao, Y.-G. Li, L. Brassart, Pressure-sensitive plasticity of lithiated silicon in Li-ion batteries, Acta Mech. Sinica 29 (2013) 379-87.

[84] B. W. Sheldon, S. K. Soni, X. Xiao, Y. Qi, Stress contributions to solution thermodynamics in Li-Si alloys, Electrochem. Solid-State Lett. 15 (2012) A9.

[85] J. Pan, Q. Zhang, J. Li, M. J. Beck, X. Xiao, Y.-T. Cheng, Effects of stress on lithium transport in amorphous silicon electrodes for lithium-ion batteries, Nano Energy 13 (2015) 192-9.

[86] H. Yang, W. Liang, X. Guo, C.-M. Wang, S. Zhang, Strong kinetics-stress coupling in lithiation of Si and Ge anodes, Extreme Mech. Lett. 2 (2015) 1-6.

[87] V. A. Sethuraman, V. Srinivasan, A. F. Bower, P. R. Guduru, In situ measurements of stress-potential coupling in lithiated silicon, J. Electrochem. Soc. 157 (2010) A1253.

[88] M. Gu, H. Yang, D. E. Perea, J. G. Zhang, S. Zhang, C. M. Wang, Bending-induced symmetry breaking of lithiation in germanium nanowires, Nano Lett. 14 (2014) $4622-7$.

[89] G. Sandu, L. Brassart, J.-F. Gohy, T. Pardoen, S. Melinte, A. Vlad, Surface coating mediated swelling and fracture of silicon nanowires during lithiation, ACS Nano 8 (2014) 9427-36.

[90] L. Luo, P. Zhao, H. Yang, B. Liu, J. G. Zhang, Y. Cui, G. Yu, S. Zhang, C. M. Wang, Surface coating constraint induced self-discharging of silicon nanoparticles as anodes for lithium ion batteries, Nano Lett. 15 (2015) 7016-22.

[91] K. Zhao, G. A. Tritsaris, M. Pharr, W. L. Wang, O. Okeke, Z. Suo, J. J. Vlassak, E. Kaxiras, Reactive flow in silicon electrodes assisted by the insertion of lithium, Nano Lett. 12 (2012) 4397-403. 
[92] V. A. Sethuraman, M. J. Chon, M. Shimshak, N. Van Winkle, P. R. Guduru, In situ measurement of biaxial modulus of Si anode for Li-ion batteries, Electrochem. Commun. 12 (2010) 1614-7.

[93] V. Shenoy, P. Johari, Y. Qi, Elastic softening of amorphous and crystalline Li-Si phases with increasing Li concentration: A first-principles study, J. Power Sources 195 (2010) 6825-30.

[94] Z. Zeng, N. Liu, Q. Zeng, Y. Ding, S. Qu, Y. Cui, W. L. Mao, Elastic moduli of polycrystalline $\mathrm{Li}_{15} \mathrm{Si}_{4}$ produced in lithium ion batteries, J. Power Sources 242 (2013) 7325.

[95] B. Hertzberg, J. Benson, G. Yushin, Ex-situ depth-sensing indentation measurements of electrochemically produced Si-Li alloy films, Electrochem. Commun. 13 (2011) 818-21.

[96] Y. S. Choi, M. Pharr, K. H. Oh, J. J. Vlassak, A simple technique for measuring the fracture energy of lithiated thin-film silicon electrodes at various lithium concentrations, J. Power Sources 294 (2015) 159-66.

[97] J. B. Ratchford, B. A. Crawford, J. Wolfenstine, J. L. Allen, C. A. Lundgren, Young's modulus of polycrystalline $\mathrm{Li}_{12} \mathrm{Si}_{7}$ using nanoindentation testing, J. Power Sources 211 (2012) 1-3.

[98] J. B. Ratchford, B. E. Schuster, B. A. Crawford, C. A. Lundgren, J. L. Allen, J. Wolfenstine, Young's modulus of polycrystalline $\mathrm{Li}_{22} \mathrm{Si}_{5}$, J. Power Sources 196 (2011) 7747-9.

[99] A.-H. Zinn, S. Borhani-Haghighi, E. Ventosa, J. Pfetzing-Micklich, N. Wieczorek, W. Schuhmann, A. Ludwig, Mechanical properties of $\mathrm{SiLi}_{\mathrm{x}}$ thin films at different stages of electrochemical Li insertion, Phys.StatusSolidi A 211 (2014) 2650-6.

[100] L. A. Berla, S. W. Lee, Y. Cui, W. D. Nix, Mechanical behavior of electrochemically lithiated silicon, J. Power Sources 273 (2015) 41-51.

[101] X. Wang, F. Fan, J. Wang, H. Wang, S. Tao, A. Yang, Y. Liu, H. B. Chew, S. X. Mao, T. Zhu, S. Xia, High damage tolerance of electrochemically lithiated silicon, Nat.Commun. 6 (2015).

[102] Y. Qi, L. G. Hector, C. James, K. J. Kim, Lithium concentration dependent elastic properties of battery electrode materials from first principles calculations, J. Electrochem. Soc. 161 (2014) F3010-F8. 
[103] A. Kushima, J. Y. Huang, J. Li, Quantitative fracture strength and plasticity measurements of lithiated silicon nanowires by in situ TEM tensile experiments, ACS Nano 6 (2012) 9425-32.

[104] S. T. Boles, C. V. Thompson, O. Kraft, R. Mönig, In situ tensile and creep testing of lithiated silicon nanowires, Appl. Phys. Lett. 103 (2013) 263906.

[105] M. Pharr, Z. Suo, J. J. Vlassak, Measurements of the fracture energy of lithiated silicon electrodes of Li-ion batteries, Nano Lett. 13 (2013) 5570-7.

[106] S. P. Nadimpalli, V. A. Sethuraman, G. Bucci, V. Srinivasan, A. F. Bower, P. R. Guduru, On plastic deformation and fracture in $\mathrm{Si}$ films during electrochemical lithiation/delithiation cycling, J. Electrochem. Soc. 160 (2013) A1885-A93.

[107] B. Ding, X. Li, X. Zhang, H. Wu, Z. Xu, H. Gao, Brittle versus ductile fracture mechanism transition in amorphous lithiated silicon: From intrinsic nanoscale cavitation to shear banding, Nano Energy 18 (2015) 89-96.

[108] X. Wang, A. Yang, S. Xia, Fracture toughness characterization of lithiated germanium as an anode material for lithium-ion batteries, J. Electrochem. Soc. 163 (2016) A90-A5.

[109] S. Lee, J. Yang, W. Lu, Debonding at the interface between active particles and PVDF binder in Li-ion batteries, Extreme Mech. Lett. 6 (2016) 37-44.

[110] M. E. Stournara, X. Xiao, Y. Qi, P. Johari, P. Lu, B. W. Sheldon, H. Gao, V. B. Shenoy, Li segregation induces structure and strength changes at the amorphous $\mathrm{Si} / \mathrm{Cu}$ interface, Nano Lett. 13 (2013) 4759-68.

[111] H. Wang, B. Hou, X. Wang, S. Xia, H. B. Chew, Atomic-scale mechanisms of sliding along an interdiffused Li-Si-Cu interface, Nano Lett. 15 (2015) 1716-21.

[112] F. Xia, S. Kwon, W. W. Lee, Z. Liu, S. Kim, T. Song, K. J. Choi, U. Paik, W. I. Park, Graphene as an interfacial layer for improving cycling performance of Si nanowires in lithium-ion batteries, Nano Lett. 15 (2015) 6658-64.

[113] S. W. Lee, H. W. Lee, I. Ryu, W. D. Nix, H. Gao, Y. Cui, Kinetics and fracture resistance of lithiated silicon nanostructure pairs controlled by their mechanical interaction, Nat. Commun. 6 (2015) 7533.

[114] R. Xu, K. Zhao, Mechanical interactions regulated kinetics and morphology of composite electrodes in Li-ion batteries, Extreme Mech. Lett. (2015) DOI: 10.1016/j.eml.2015.10.004. 
[115] E. M. C. Jones, M. N. Silberstein, S. R. White, N. R. Sottos, In situ measurements of strains in composite battery electrodes during electrochemical cycling, Experim. Mech. 54 (2014) 971-85.

[116] S. A. Roberts, V. E. Brunini, K. N. Long, A. M. Grillet, A framework for threedimensional mesoscale modeling of anisotropic swelling and mechanical deformation in lithium-ion electrodes, J. Electrochem. Soc. 161 (2014) F3052-F9.

[117] S. P. V. Nadimpalli, V. A. Sethuraman, D. P. Abraham, A. F. Bower, P. R. Guduru, Stress evolution in lithium-ion composite electrodes during electrochemical cycling and resulting internal pressures on the cell casing, J. Electrochem. Soc. 162 (2015) A2656-A63.

[118] D. Y. W. Yu, M. Zhao, H. E. Hoster, Suppressing vertical displacement of lithiated silicon particles in high volumetric capacity battery electrodes, ChemElectroChem 2 (2015) 10905.

[119] X. Xiao, W. Zhou, Y. Kim, I. Ryu, M. Gu, C. Wang, G. Liu, Z. Liu, H. Gao, Regulated breathing effect of silicon negative electrode for dramatically enhanced performance of Li-ion battery, Adv. Func. Mater. 25 (2015) 1426-33.

[120] V. A. Sethuraman, A. Nguyen, M. J. Chon, S. P. V. Nadimpalli, H. Wang, D. P. Abraham, A. F. Bower, V. B. Shenoy, P. R. Guduru, Stress evolution in composite silicon electrodes during lithiation/delithiation, J. Electrochem. Soc. 160 (2013) A739-A46.

[121] V. Kuznetsov, A. H. Zinn, G. Zampardi, S. Borhani-Haghighi, F. La Mantia, A. Ludwig, W. Schuhmann, E. Ventosa, Wet nanoindentation of the solid electrolyte interphase on thin film Si electrodes, ACS Appl. Mater. Interfaces 7 (2015) 23554-63.

[122] J. Zheng, H. Zheng, R. Wang, L. Ben, W. Lu, L. Chen, L. Chen, H. Li, 3D visualization of inhomogeneous multi-layered structure and Young's modulus of the solid electrolyte interphase (SEI) on silicon anodes for lithium ion batteries, Phys. Chem. Chem. Phys. 16 (2014) 13229-38.

[123] A. Tokranov, R. Kumar, C. Li, S. Minne, X. Xiao, B. W. Sheldon, Control and optimization of the electrochemical and mechanical properties of the solid electrolyte interphase on silicon electrodes in lithium ion batteries, Adv. Energy Mater. (2016) DOI: 10.1002/aenm.201502302. 
[124] Z. Zeng, N. Liu, Q. Zeng, S. W. Lee, W. L. Mao, Y. Cui, In situ measurement of lithiationinduced stress in silicon nanoparticles using micro-raman spectroscopy, Nano Energy 22 (2016) 105-10. 